\title{
THE OBSERVATION OF LAND RESOURCES FROM SPACE
}

\author{
Paul M. Mather \\ Department of Geography \\ University of Nottingham
}

\section{INTRODUCTION}

Satellite-borne sensors are now routinely observing the Earth's land and sea surfaces in a range of wavebands and at a variety of spatial scales. The best-known of these systems, carried by Landsat, has been operating since 1972; more recently, the French SPOT and the Japanese MOS programmes have provided high-resolution data in the visible and infrared regions, while coarse-resolution imagery has continued to be supplied by AVHRR and the Meteosat and related geostationary satellite systems. The launch in 1991 of the European ERS-1 and the Japanese JERS-1 provided imaging radar data for the first time since Seasat in 1978. Plans for the next 10 years include:

- ERS-2 (1993), which will carry a Synthetic Aperture Radar (SAR) similar to the ERS-1 SAR, plus a visible and infrared narrow-band scanner (ATSR-2),

- NOAA 'I' - 'M' (1993-1996), carrying the Advanced Very High Resolution Radiometer-2 and -3 (AVHRR-2/-3),

- Landsat-6 (1993), which will have an Enhanced Thematic Mapper (ETM) instrument,

- SPOT-3 (1993), with the High Resolution Visible (HRV) sensor equivalent to SPOT-1 and -2 ,

- SPOT-4 (1996), carrying an enhanced HRV termed the HRVIV or High Resolution Visible and Infrared sensor, plus the Vegetation Sensor, and

- Radarsat (1995), which will have a Synthetic Aperture Radar.

ESA is planning for the launch of the first Polar Orbit Earth Observation Mission (POEM) in 1997. There are two separate sensor packages planned for POEM: 
the $M$ series, which are primarily meteorological, oceanographic and climatological, and the $N$ series oriented towards Earth resources and atmosphere. The first European Polar Platform, EPOP-1, will carry the $M$ series sensor package, including VIRSR (similar to AVHRR), a Synthetic Aperture Radar, MERIS (a medium resolution imaging spectrometer designed for ocean applications), and AATSR (advanced along-track scanning radiometer, designed primarily for sea-surface temperature studies), in addition to atmospheric sounding devices and a radar altimeter (Rast \& Readings, 1992). NASA is planning a parallel system, called EOS (Earth Observing System) for launch in 1998. Plans for EOS and its associated Data and Information System, EOSDIS, are in a state of flux at the time of writing.

The motivation underlying this remarkable expansion of Earth observation capabilities during the next ten years is primarily the perceived need to improve our understanding of the Earth system as a whole, including human activity. Rast \& Readings (1992) list the four themes which underlie ESA's Earth Observation programme, as follows:

- monitoring the Earth's environment on various scales, from local to regional to global,

- management and monitoring of the Earth's resources, both renewable and non-renewable,

- continuing provision of an operational meteorological system, and

- improving scientific knowledge of the structure and dynamics of the Earth's crust and interior.

Rast \& Readings comment as follows: "More organised information about the behaviour of the environment and factors influencing the Earth's natural resources can only be achieved on the basis of a better understanding of the Earth when viewed as a system ..... Fundamental to this is the provision of data to identify processes and to validate models." (1992, p.175-176). Clearly, these authors view the provision of Earth observation data in terms of the classical process of scientific explanation, which requires hypotheses or models to be established and subsequently tested against observational data. This procedure implies that observational data have the following characteristics:

- to be internally consistent,

- to relate to other observations in a known manner,

- to be readily available to researchers.

If Earth observation data are to have any scientific value they must satisfy these three criteria. The purpose of this paper is to examine the obstacles that presently stand in the way of this ideal, and to propose mechanisms that will ensure that Earth observation data of a sufficient quality are readily available to researchers. 
Given that the first two of ESA's policy themes, listed above, are concerned with global problems, it is essential to ensure that researchers have access to global data sets.

\section{OBSTACLES TO THE SCIENTIFIC USE OF EARTH OBSERVATION DATA}

Earth observation (EO) data are provided to the user on magnetic tape in the form of digital images, which are composed of registered arrays of numbers on a 0 255 or $0-1024$ scale. These numbers are quantised counts, and are not immediately related to physical quantities such as radiant flux. Methods of processing these data, and extracting information from them, are widely known (Chuvieco, 1990; Mather, 1987). Generally speaking, such methods involve the enhancement of a single-date image using digital image processing techniques such as contrast improvement or filtering, or the derivation of map-like products using pattern recognition or classification techniques. It is important to realise that the data forming the image set are related only in a loose way to physical properties of the environment. Furthermore, these relationships may vary over the image, depending on topography and atmosphere. It is unlikely that the same relationships would hold at a different point in time. EO data, in their raw form, are therefore internally and externally inconsistent, and consequently require pre-processing before they are suitable to input to predictive models, such as Running \& Coughlan's (1988) forest ecosystem process model, the Biosphere-Atmosphere Transfer Scheme Dickinson, (1984) and the simple biosphere model, Sellers \& Dorman (1987).

The signal received by a sensor operating in the visible and near infrared wavebands of the electromagnetic spectrum is often represented in simple terms as the solar spectrum modified by interaction with the atmosphere and with the ground surface. However, these interactions are more complex, as explained by Duggin \& Robinove (1991). Their paper discusses eleven major assumptions that are implicit in the acquisition and analysis of passively-sensed digital image data. These authors identify the following factors, among others, as relating to the correlation between ground attributes in the real world and the corresponding properties inferred from remotely-sensed data: (i) nature of the material forming the ground pixel area, (ii) correlation of the attributes of this area with the upwelling spectral radiance field, (iii) effects of atmospheric attenuation and scattering, (iv) performance of the analogue-to-digital converter (affecting the response, calibration and linearity of the sensor), (v) influence of any data compression/decompression, and (vi) choice of image processing or pattern recognition procedures. Other factors refer to the relationship between the sensor attributes, such as resolution, spectral bands, and spectral response, and to the properties of the target of interest. Duggin and Robinove express the problem succinctly as follows: "Fundamental implicit assumptions in image acquisition and analysis are that the radiance properties recorded on the image represent the optical properties of those features on the ground which are of interest and that the image, 
after processing, may be rectified and superimposed on a map to represent accurately the ground features which are of interest" (1990, p. 1673).

In order to ensure that image data are internally consistent prior to analysis, preprocessing is necessary in order to remove, as far as possible, the 'external' effects recognised in the previous paragraphs. Such preprocessing relates to the need for sensor calibration, correction for atmospheric, illumination and viewing geometry effects, and geo-referencing. Geo-referencing is not strictly speaking a preprocessing operation, in that it is not expressly concerned with the removal or attenuation of a sensor-imposed property; rather, it refers to the registration of the image to an accepted map projection. In the sense that such an operation is necessary for the use of GIS, it will be considered at this point.

\section{RADIOMETRIC CALIBRATION}

Passive imaging sensors, carried by orbiting satellites, collect upwelling radiance from the Earth's surface in one or more spectral bands for each of a large number of ground cells or pixels. The output for each spectral band from the sensor is a voltage, which is proportional to the spectral radiance; the relationship between voltage and spectral radiance is approximately linear. Asrar (1989) shows that the relationship relies on the gain and offset of the sensor system. These values are found initially from pre-launch ground calibration and subsequently by in-flight calibration or by reference to ground targets of known reflectance. Gain and offset values are normally recorded in the header file of the data tapes. The quantised counts contained on the tape can be converted to radiances by reference to the gain and offset for the given spectral band. Since the sensor gains and offsets vary over the life of the sensor, it follows that the raw pixel value cannot have any absolute meaning. Thus, a pixel value of 60 in a certain spectral band may be said to represent a lower radiance than a value of 50 in another pixel in the same image, other things being equal, but we cannot make any judgement as to its relationship to a value of 50 measured by the same sensor at a different time. It also follows that inferences regarding relative magnitudes of pixel values obtained by different sensors, such as HRV or TM, cannot be made unless the quantised counts are converted first to radiances.

\section{ATMOSPHERIC CORRECTION}

Solar radiation incident upon the Earth's surface must first pass through the atmosphere. Equally, reflected or emitted radiation passes upwards through the atmosphere to be intercepted by the sensor system. The spectral composition of the signal is modified by interactions with the atmosphere on both the upward and the downward journeys. The atmosphere is not, unfortunately, constant in its composition either in space or in time, and consequently changes in the level of electromagnetic radiation recorded by the sensor for a given area cannot, even after 
radiometric correction, be presumed to indicate changes in the ground area being observed. Kaufman (1989) notes the following effects that are generated by the atmospheric effect: variation of the severity of the effect with wavelength, which may affect discrimination between stressed and unstressed vegetation; alteration i the spatial distribution of reflected radiation, affecting the spatial resolution of the system; changes in the apparent brightness of a target, affecting measurements of albedo and reflection; and generation of spatial variations in apparent surface reflection through the effect of sub-pixel sized clouds. Correction for atmospheric effects is difficult, because the optical characteristics of the atmosphere (which are influenced by aerosol optical thickness, phase function, single-scattering albedo, gaseous absorption, and vertical profile) are rarely known in any detail at the time of imaging and over the area of the image. Atmospheric models such as LOWTRAN (Kneisys et al., 1983) provide corrections based on theory, using either the observed or inferred characteristics of the atmosphere (the 'standard atmospheres', such as maritime temperate winter), but such approximations are correct only to the first order, at best. Hill and Sturm (1988) use a basic 'histogram minimum' method (USGS, 1979) to estimate atmospheric path radiance, followed by correction for absorption based on LOWTRAN functions. The removal of the effects on the spectral properties of the signal caused by atmospheric interactions remains one of the fundamental problems of remote sensing; without a reliable and satisfactory method of correction the recovery of upwelling radiances, and hence the credible comparison of spectra across images, will remain problematical.

\section{ILLUMINATION AND VIEWING GEOMETRY EFFECTS}

Most natural terrestrial surfaces have reflectance spectra which depend upon the angle of view and the angle of illumination, relative to the ground surface. The distribution of reflected energy as a function of the angles of incidence and reflection is described by the hemispherical bidirectional reflectance function of the target, which is generally unknown. If the surface is assumed to be Lambertian then a correction for solar illumination angle can be made, but this is only an approximation. Viewing angle depends on the properties of the satellite orbit and the characteristics of the sensor. For example, it would be realistic to suggest that the same object viewed by the AVHRR at either side of the swath $\left( \pm 55^{\circ}\right)$ and at nadir would produce three different values, and a similar effect might be achieved by viewing the same area at different HRV tilt angles (HRV can be tilted up to $\pm 23^{\circ}$ from nadir). It is therefore the case that a homogeneous cover, such as an agricultural crop growing on a flat area, will produce a variable spatial distribution of radiance in a specific spectral band, the variation depending on scan and illumination angles (Moran et al., 1990, Royer et al., 1985). Over calm water, the effect can be to produce 'sun glitter', which is common on AVHRR images. The effects of terrain must be added to the bidirectional reflectance characteristics of the target. It is well known that the topographic position of the ground cell, that is, its slope and aspect, have a significant effect on the characteristics of the signal emanating from that cell (e.g. Holben \& Justice (1980) conclude that: " ... the 
topographic effect ... can produce a wide variation in the radiances associated with a given land-cover type"). In hilly or mountainous areas, the variation in spectral reflectance for targets of the same land cover type can be considerable. Where a Digital Elevation Model is available, a first-order correction can be applied to remove the primary effects of slope and aspect (Jones et al., 1988, Thomson \& Jones, 1990).

\section{GEO-REFERENCING}

Information derived from remotely-sensed data is increasingly being used within a Geographical Information System. In some instances, for example the correction for terrain effects which is discussed in the preceding paragraph, information from a GIS is used in the correction of images. In other instances, such as knowledge-based classification, map and other data are used in parallel with image data in the information-extraction process. In order for such operations to be possible, both map and image must be represented using the same coordinate system. Normally, this correspondence is achieved by the geometric correction of the image. Methods of geometric correction based on the computation of leastsquares bivariate polynomials of low order are well known (e.g. Mather, 1987). The sensitivity of the method to variations in sample size and in the spatial distribution of ground control points is perhaps not as widely appreciated; a recent study (Mather, 1992) provides some interesting results, which show that the accuracy and precision of the estimates of the polynomial coefficients drops significantly as the ground control point distribution changes from random to linear. The effect of reducing the sample size is shown to result in a lower precision. These factors should be taken into consideration otherwise the geographic correspondence between map and image will be lost.

\section{ACCESS TO DATA}

The foregoing sections are concerned with ensuring the quality of remotelysensed data prior to their use in modelling studies or in change detection. Quality is a somewhat indeterminate property, since its definition depends primarily on the uses to which the data are to be put. Nevertheless, attention must be paid to data quality aspects if remotely-sensed data are to contribute properly to environmental studies and to studies of global change. Such studies often involve inter- and multidisciplinary groups which may not have primary expertise in remote sensing, hence a factor as important as data quality (i.e. suitability of the data for the intended use) is awareness of the existence of the data, their properties, and the information that can be derived from them. In recent months, several developments have occurred which demonstrate the importance of raising the awareness of the environmental research community of the existence of suitable remotely-sensed data. The first of these developments is GENIE (Global Environmental Network for Information Exchange). This project is based at the Universities of Loughborough and 
Nottingham, England, and is concerned with the establishment and maintenance of a metadata system. Metadata are descriptions of data, including location, format, nature (such as thermal infrared or SAR), and ordering information. GENIE is intended to go further than this, and -given a description of the intended use of the data- provide details of preprocessing and information-extraction methods that may be used with the specified data set. A naive user will, using GENIE, be able to request information about the availability of data for a specified application and for a specific geographical area. GENIE will respond with information pertinent to the scale and the information requirements of the user and, with additional prompting, will detail the various operations (and the pitfalls) involved in using the data. A further development of GENIE will be the inclusion of descriptions of appropriate algorithms which have been tested and verified. The kind of algorithm that will be referenced by GENIE will be those that are not normally found in standard image processing systems but which are developed and used by the research community, such as scan angle corrections, It is expected that GENIE will be available in experimental mode in 1993.

The European Economic Community (EEC) and the European Space Agency (ESA) have announced their intention to establish a Centre for Earth Observation (CEO) and are currently at the stage of commissioning a feasibility study. CEO is meant to ensure the long-term supply and maintenance of $E O$ and derived environmental data, and thus will have cataloguing, archiving and processing capabilities. It will comprise a decentralised network, coordinated by ESRIN (ESA) and the JRC (CEC). A preliminary feasibility study for a Global Environmental Network Information and User System (GENIUS) was completed by ESA in 1991. CEO will complement developments taking place elsewhere, particularly in the United States (where the EOSDIS programme is already established, though currently it is experiencing financial and managerial problems) and Japan. It is not difficult to see that GENIE would provide an ideal front-end to the CEO operation.

These developments represent the response of national and supra-national government agencies to the challenge of providing global change researchers with access to data (and metadata) in order that their scientific studies may be more firmly based. The challenge to the remote sensing community is to ensure that the quality of the data and information supplied via these networks is adequate. For this aim to be achieved, considerable attention must be given to the preprocessing of data and to the methods used in information extraction. The empirical methods used in the $1970 \mathrm{~s}$ and 1980 s will no longer suffice. These methods served their purpose by demonstrating the capability of remote sensing to provide otherwise unobtainable environmental information. Now, to quote Dr Garret FitzGerald, former prime Minister of Ireland, "It works in practice. Now let's try and make it work in theory.".

\section{REFERENCES}

ASRAR, G. (ed.) (1989): Theory and applications of optical remote sensing. John Wiley, New York. 
CHUVIECO, E. (1990): Fundamentos de teledeteccion espacial. Ediciones Rialp, S.A. Madrid.

DICKINSON, R.E. (1984): "Modelling evapotranspiration for three-dimensional global climate models". Geophysics Monographs, American Geophysical Union , 29, 58-72.

DUGGIN, M.J. \& ROBINOVE, C.J. (1990): "Assumptions implicit in remote sensing data acquisition and analysis". International Journal of Remote Sensing, 11, 1669-1694.

Hill, J. \& STURM, B. (1988): "Radiometric normalisation of multi-temporal Thematic Mapper data for the use of greenness profiles in agricultural landcover classification and vegetation monitoring". In Proceedings of the EARSeL 8th Symposium: Alpine and Mediterranean Areas, held in Capri, Italy, 17-20 May 1988, 21-40.

HOLBEN, B.N. \& JUSTICE, C.O. (1980): "The topographic effect on spectral response of nadir-pointing sensors". Photogrammetric Engineering and Remote Sensing, 46, 1191-1200.

JONES, A.R., SETTLE, J.J. \& WYATT, B.K. (1988): "Use of digital terrain data in the interpretation of SPOT HRV data". International Journal of Remote Sensing, 9, 669-682.

KAUFMAN, Y. (1989): "The atmospheric effect on remote sensing and its correction". In Asrar, G. (ed.), op. cit., 336-428.

KNEISYS, F.X., SHETTLE, E.P., GALLERY, W.O., CHETWYND, J.H., ABREU, L.W., SELBY, J.E.A., CLOUGH, S.A. \& FENN, R.W. (1983): Atmospheric transmittance/radiance: computer code LOWTRAN 6. AFGL-TR-83-0187, Air Force Geophysics Laboratory, Hanscom Air Force Base, Massachusetts.

MATHER, P.M. (1987): Computer processing of remotely-sensed images: an introduction. John Wiley \& Sons, Chichester. (Reprinted 1989).

MATHER, P.M. (1992): "Geometric correction using least squares: a sensitivity analysis". In: Remote sensing - from research to operations. Proceedings of the 1992 Annual Conference of The Remote Sensing Society, Dundee, September 1992. The Remote Sensing Society, Nottingham (forthcoming).

MORAN, M.S., JACKSON, R.D., HART, G.F., SLATER, P.N., BARTELL, R.J., BIGGAR, S.F., GELLMAN, D.I. \& SANTER, R.P. (1990): "Obtaining surface reflectance factors from atmospheric and view-angle corrected SPOT-1 HRV data". Remote Sensing of Environment, 32, 203-214.

RAST, M. \& READINGS, C.J. (1992): "The ESA Earth Observation Polar Platform Programme". In Mather, P.M. (ed.): TERRA-1: Understanding the terrestrial environment - The role of Earth observations from space. Taylor \& Francis, London, p.175-184.

ROYER, A., VINCENT, P. \& BONN, F. (1985): "Evaluation and correction of viewing angle effects on satellite measurements of bidirectional reflectance". Photogrammetric Engineering and Remote Sensing, 51, 1899-1914.

RUNNING, S.W. \& COUGHLAN, J.C. (1988): "A general model of forest ecosystem processes for regional applications. I: Hydrological balance, canopy gas exchange and primary production processes". Ecological Modelling, 42, 125-154.

SELLERS, P.J. \& DORMAN, J.L. (1987): "Testing the simple biosphere model (SiB) using point micrometeorological and biophysical data". Journal of Climatology and Applied Meteorology, 26,

THOMSON, A.G. \& JONES, C. (1990): "Effects of topography on radiance from upland vegetation in North Wales". International Journal of Remote Sensing, 11, 829-840.

USGS (1979): Landsat data users handbook. Eros Data Center, Sioux Falls, South Dakota, USA. 